\title{
Capsule Commentary on Edelman et al., Shared Medical Appointments for Patients with Diabetes Mellitus: A Systematic Review
}

\author{
Kristina P. Schumann, PhD \\ VA Connecticut Healthcare System, West Haven, USA.
}

J Gen Intern Med 30(1):97

DOI: $10.1007 / \mathrm{s} 11606-014-3042-3$

(c) Society of General Internal Medicine 2014

$\mathrm{T}$ his systematic review by Edelman et al. ${ }^{1}$ explores shared medical appointments (SMAs) as a tool for improving access to and quality of care for diabetes management. SMAs were effective in reducing hemoglobin A1c and systolic blood pressure, but did not lower LDL cholesterol. Understanding of the effects of SMAs on nonbiophysical outcomes (e.g., hospital admissions, costs, patient satisfaction, self-management behaviors, etc.) was limited by significant heterogeneity among the studies. Additionally, the authors were unable to draw conclusions about characteristics of SMAs that lead to successful outcomes (e.g., number of sessions, closed vs. open groups, etc.), due to heterogeneity in SMA design. The authors conclude that SMAs are promising, but future research should focus on consistent measurement of nonbiophysical outcomes and a clearer understanding of the effective components of SMAs.

SMAs often include an educational component in addition to medication management. ${ }^{2}$ This review demonstrates that, although most SMAs included in the review had an educational component, researchers focused on biophysical outcomes (specifically A1c, BP, and cholesterol) with relatively little attention paid to the effectiveness of SMAs on providing the necessary education and behavioral interventions. The American Association of Diabetes Educators (AADE) identifies seven key self-care behaviors (AADE 7) (i.e., healthy eating, physical activity, medication adherence, selfmonitoring of blood glucose, problem solving, reducing risks/complications, and coping) as essential in diabetes management, and highlights the importance of intervening in these behaviors to positively impact biophysical outcomes. ${ }^{3}$ Future research on SMAs should consistently measure the effectiveness of SMAs in improving the AADE 7 behaviors.

SMAs show promise in reducing costs and increasing efficiency for healthcare providers. ${ }^{4}$ SMAs can offer patients a cost-effective way to benefit from interventions provided by a variety of disciplines (e.g., medicine, pharmacy, nursing, mental health, nutrition, physical therapy, etc.). Future research should focus on understanding how to maximize the contribution of each discipline to enhance the patient experience and improve disease outcomes. Future research should focus on understanding how and why SMAs are effective, which patients they are most/least effective for, and what can be done to further improve this novel approach to patient care.

Conflict of Interest: The author has no conflicts with this manuscript.

Corresponding Author: Kristina P. Schumann, PhD; VA Connecticut Healthcare System, West Haven, USA (e-mail: Kris.schumann@gmail.com).

\section{REFERENCES}

1. Edelman D, Gierisch JM, McDuffie JR, Oddone E, Williams JW. Shared medical appointments for patients with diabetes mellitus: A systematic review. J Gen Int Med. 2014. doi:10.1007/s11606-0142978-7.

2. Weinger K. Group medical appointments in diabetes care: Is there a future? Diabetes Spectr. 2003;16(2): 104-107.

3. American Association of Diabetes Educators. Standards for outcomes measurement of diabetes self-management education. Diabetes Educ. 2003;29(5):804-816.

4. Sanchez I. Implementation of a diabetes self-management education program in primary care for adults using shared medical appointments. Diabetes Educ. 2011;37(3):381-391. 